\title{
Medicamentos, cantidad y calidad
}

\section{Medicines, quantity and quality}

\section{E. Sempere}

Cuando se habla de uso de medicamentos se plantean inevitablemente dos cuestiones relacionadas: 1 . ¿Se utilizan demasiados medicamentos? 2. ¿Se utilizan bien?

1. Aunque la opinión de la población general, e incluso de gran parte del colectivo médico, es que se deben utilizar todos los que sean necesarios, es evidente que el crecimiento en el consumo de medicamentos debe ser, como todas las actividades humanas, "sostenible", y a tenor de los datos del último informe del Ministerio de Sanidad y Consumo es evidente que en el Estado español no lo es ${ }^{1}$. En 2006 España dedicó a medicamentos el 21,3\% del gasto sanitario total, el más elevado de los países integrados en la OCDE. Si analizamos los datos de estos países, se comprueban grandes diferencias en el gasto farmacéutico, pudiéndose distinguir tres patrones básicos:

- El de algunos países escandinavos (Noruega, y Dinamarca), Suiza y EEUU con porcentajes cercanos o inferiores al $10 \%$ del gasto sanitario total.

- Países centroeuropeos (Francia, Alemania), Suecia, Finlandia y Canadá, con porcentajes entre el 10 y el $15 \%$.

- Países del sur de Europa (Italia, España y Portugal) con porcentajes superiores al $20 \%$.

La mayoría de estos países han conseguido reducir en los últimos años este porcentaje, aunque España ha sido el país que menos éxito ha tenido. Es decir, que gastamos demasiado dinero en medicamentos y por tanto mucho menos en otros recursos sanitarios.

Otra dato diferente es el del crecimiento interanual en medicamentos. Dato bastante más aireado por la administración sanitaria, sobre todo por la necesidad de su contención, aunque no es el determinante último del gasto en medicamentos. Así por ejemplo, los EEUU tienen un crecimiento interanual en medicamentos muy superior al $10 \%$, casi el doble del de España, mientras que el porcentaje del gasto sanitario que dedica a ellos, como hemos visto, es menos de la mitad del

\section{Correspondencia:}

Ermengol Sempere Verdú

Centro de Salud de Paterna (Valencia)

Agencia Valencia de Salud

Grupo de Utilización de Fármacos de la

Sociedad Española de Medicina Familiar y

Comunitaria

E-mail: mere@comv.es 
Español. Ante las perspectivas económicas nada halagüeñas, España está obligada a frenar el consumo de recursos inadecuados, incluido el de fármacos, para lo cual se deberían fijar prioridades basadas en criterios de eficiencia de salud pública.

En lo referente a la Comunidad Autónoma de Navarra, según los datos de gasto farmacéutico de 2008, fue la segunda comunidad autónoma con menor incremento interanual, situándose en el 5,98\%, por debajo del $6,87 \%$ estatal, y a cierta distancia de la Comunidad Autónoma que obtuvo el menor incremento, la de Madrid con el $5,2 \%$. El gasto en medicamentos por habitante fue de $254 € /$ año, por debajo de la media estatal, con un porcentaje de prescripciones de genéricos del $16,2 \%$, ligeramente por debajo de la media estatal del $17,8 \%^{2}$. En resumen, el gasto en medicamentos de la CA de Navarra es sensiblemente menor que el de la media estatal, con un menor gasto medio por habitante. Esta moderación no parece estar relacionada con el consumo de genéricos, que está por debajo de la media estatal, ni por el número de recetas por habitante, que está muy cerca de la media estatal, sino por la reducción experimentada en el período 2004-2007 del precio medio por receta, que fue del $-5,82 \%$.

2. En cuanto a la pregunta de si los fármacos se prescriben bien o no, los datos aportados por el estudio de Garjón ${ }^{3}$ indican que, al menos en la CA de Navarra, hay aspectos sensiblemente mejorables. Atendiendo a la información generada por las recetas facturadas durante un mes de 2008 por el Servicio Navarro de Salud-Osasunbidea, el autor subraya los siguientes aspectos mejorables:

- La polifarmacia, al detectar que el 3,4\% de los navarros retiró al menos diez medicamentos distintos durante un mes.

- El uso desaconsejable o contraindicado de determinadas combinaciones, sobre todo de AINE y de medicamentos con acción anticolinérgica.

- El posible sobreuso de los inhibidores de la bomba de protones (prescrito al 11\% de la población navarra).

- El abuso de los psicotropos en ancianos (el 6,2\% de los ancianos consumía tres o más, y el $24,3 \%$ al menos una benzodiacepina).

- La dependencia a las benzododiacepinas (el 82,4\% de sus usuarios la utilizaban al menos durante ocho meses).

Los datos son de sumo interés y apoyan una idea de una determinada cultura del medicamento, compartida posiblemente por médicos y pacientes, de que casi todo se puede tratar con medicamentos ("a pill for every ill"), del uso indiscriminado de los AINE como "matadolor" universal y del tratamiento irreflexivo de los trastornos emocionales con medicamentos que acaban por generar dependencia, al margen de la duración del proceso que generó la prescripción.

No obstante, son datos aislados de las referencias sobre las enfermedades y las personas que los padecen y sería interesante disponer de datos comparativos de otras Comunidades Autónomas.

A esta lista de datos preocupantes de prescripción, convendría añadir algunos de todo el Estado Español y en general de las sociedades desarrolladas, como es el aumento de la incidencia de los problemas y reacciones adversas a medicamentos ${ }^{4}$, el imparable aumento en el consumo de antidepresivos ${ }^{5}$, el excesivo consumo de antibióticos, con un elevado porcentaje de los de amplio espectro ${ }^{6}$, el uso cada vez mayor de bifosfonatos ${ }^{7}$, y otros medicamentos para el tratamiento de 
la osteoporosis densitométrica o el uso indiscriminado de estatinas como máximo exponente de la "riesgología".

Conviene no olvidar la necesidad de potenciar la colaboración de otros profesionales, especialmente de enfermería con su capacidad de manejo de medidas no farmacológicas y de prevención mediante actividades de educación comunitaria, hoy muy reducida en aras de la cultura del medicamento comentada.

Sobre las posibles soluciones apuntadas por el autor, figuran en primer lugar la formación, que debería estar totalmente desvinculada de la industria farmacéutica ${ }^{9}$, impulsada desde las administraciones y dirigida a todos los médicos del sistema público. Así, se deberían potenciar los contenidos específicos en uso racional del medicamento, pero también las alternativas al tratamiento farmacológico, como por ejemplo, la formación en psicoterapia, las técnicas de deshabituación de psicofármacos ${ }^{10}$, el tratamiento no farmacológico del dolor crónico, o las técnicas de motivación para promover los estilos de vida saludables. En cuanto a los posibles agentes implicados en la formación en el uso racional de medicamentos en el ámbito público, la figura del farmacólogo de departamento o de área debería ser crucial, pero su plan de formación y sus actuaciones deberían estar desvinculados de los objetivos económicos de su departamento, para evitar el rechazo de los clínicos.

El autor señala la necesidad de que cada médico disponga de sus datos de prescripción, propuesta a la que es fácil adherirse, aunque se deberían disponer tanto de los indicadores al uso de la empresa, habitualmente los requeridos para el contrato de gestión, como de los datos de auditorías autoadministradas. En cualquier caso, el acceso a la explotación de los datos de los sistemas centralizados de información sanitaria debería de estar a disposición de los profesionales, para que de una forma ágil y autónoma pudieran generar indicadores de sus pacientes, tanto cuantitativos (de prevalencia de enfermedad o de consumo de medicamentos), como cualitativos o de adecuación de sus propios tratamientos. Un modelo a seguir es el del National Precribing Service de Australia que invita a sus prescriptores a realizar de forma voluntaria sus propias auditorías mediante un sencillo manual de procedimiento ${ }^{11}$.

Respecto al uso de herramientas informáticas, cabe ser crítico con el excesivo optimismo reinante sobre las posibilidades de la historia clínica informatizada para mejorar la prescripción. Conviene recordar que los países con un mejor uso de los medicamentos lo alcanzaron mucho antes de la informatización. Sin cuestionar las inmensas posibilidades que supone el acceso a internet en la consulta, los médicos, al menos los de familia, solemos sentirnos abrumados por el exceso de alertas y de indicaciones presentes en los sistemas informáticos de las historias clínicas que interrumpen continuamente el juicio clínico y que conducen a que con elevada frecuencia se haga caso omiso de ellas. Por ejemplo, en un estudio realizado en los EEUU, los médicos de familia obviaron el $91,2 \%$ de las alertas sobre alergias y el $89,4 \%$ de las alertas sobre interacciones potencialmente graves ${ }^{12}$. Algo similar ocurrió en otro estudio en el que un sistema experto de ayuda a la prescripción no consiguió disminuir el número de problemas relacionados con medicamentos, ni siquiera los prevenibles ${ }^{13}$. Pero además, se ha podido constatar que las historias clínicas informatizadas distan mucho de ser útiles para el adecuado control clínico y por supuesto para que los indicadores de actividad y consumo y la investigación 
realizada con ellas sean mínimamente fiables en un paciente en particular, ya que con elevada frecuencia se omiten registrar datos de prescripción, alergias y sobre todo reacciones adversas confirmadas ${ }^{14}$. A pesar de este panorama, la necesidad de potenciar la seguridad del paciente obliga a la mejora continua de los sistemas de prevención de los riesgos asociados al uso de los medicamentos.

Es incuestionable el papel crucial del médico de familia en el control de las prescripciones, sin embargo su papel no es exclusivo. No se puede hablar de "dejación de funciones" el hecho de que se reclame que cada médico que prescriba a un paciente, al margen del nivel asistencial en el que desarrolle su actividad, asuma sus propias responsabilidades sobre la indicación, la pauta posológica, duración, y contraindicaciones, pero también sobre la adquisición del medicamento y la renovación de la orden de prescripción. Es inaplazable que se acometa de una vez por todas las reformas del sistema de prescripción a nivel estatal, de tal modo que se acabe con la confusión existente entre prescripción y "recetación" en el ámbito extrahospitalario, y por supuesto que se acabe con el fardo de la prescripción delegada. El médico de familia en nuestro país no puede seguir dedicando cerca de la mitad de su tiempo a generar recetas y contar envases, porque simplemente es un desperdicio de recursos. Puede que la implantación de la receta electrónica (idealmente prescripción electrónica) mejore algo esta situación, pero a condición de que sea de obligado cumplimiento para toda orden de prescripción generada por cualquier médico del sistema público ${ }^{15}$.

No hemos de olvidar, como refiere Garjón ${ }^{3}$, que existen otros agentes implicados en el uso de los medicamentos sobre los cuales se va a tener que prestar mayor atención en el futuro:

- Los profesionales de enfermería, que van a tener que asumir cada vez mayor protagonismo en el uso y control de los medicamentos, incluida la capacidad efectiva de prescripción en función de su grado de cualificación, tal y como ya existe en otros países ${ }^{16}$.

- Los farmacéuticos comunitarios, con el desarrollo de la atención farmacéutica en estrecha colaboración con los profesionales sanitarios.

- Las asociaciones de usuarios, que cada vez van a estar más informadas, y mejor organizadas.

Por último, no estaría de más recordar que el perfil de uso de medicamentos de una determinada sociedad depende de múltiples determinantes ${ }^{17}$, entre los cuales tiene un papel destacado el médico, pero también muchos otros agentes y factores, como la oferta de medicamentos y las indicaciones de uso aprobadas por las autoridades sanitarias, la información circulante sobre medicamentos (veraz, eficiente, parcial, sesgada, interesada o no), las prácticas clínicas habituales ("las costumbres clínicas"), las condiciones en la que se presta la asistencia, la calidad y eficiencia de los sistemas de registro, las expectativas de los pacientes (frecuentemente determinadas por las costumbres clínicas) y de sus grupos de presión (en ocasiones auténticos brazos armados de la industria farmacéutica), las condiciones de dispensación y los mecanismos de control de su uso. Sólo teniendo en cuenta todos estos factores será posible el uso adecuado de los medicamentos para que cumplan su objetivo de mejorar la salud de forma eficiente y saludable. 


\section{Agradecimientos}

Pilar Arroyo Anies, médico de familia, por sus comentarios y aportaciones.

\section{BIBLIOGRAFÍA}

1. Anónimo. Informe Anual del Sistema Nacional de Salud 2007. Ministerio de Sanidad y Consumo. Acceso: 28 de marzo de 2009.

http://www.actasanitaria.com/fileset/doc_48155_FICHERO_NOTICIA_65971.pdf

2. Anónimo. Gasto farmacéutico en 2008. Una evolución preocupante. Federaciones de asociaciones para la defensa de la sanidad pública (FADSP). Acceso: 28 de marzo de 2009. Disponible en: http://www.fadsp.org/pdf/presentacion_crecimiento_y_gasto_farmaceutico_09_2.doc

3. GARJón J. Prescripción de medicamentos a pacientes ambulatorios. An Sist Sanit Navar 2009; 31: 11-21.

4. Moore TJ, Cohen MR, Furberg CD. Serious adverse drug events reported to the food and drug administration, 1998-2005. Arch Intern Med 2007; 167: 1752-1759.

http://archinte.ama-assn.org/cgi/content/abstract/167/16/1752

5. Ortiz A, Lozano C. El incremento en la prescripción de antidepresivos. Aten Primaria 2005; 35: 152-155. http://www.elsevier.es/revistas/ctl_servlet?_f=7064\&articuloid=13071941

6. Cars O, Mölstad S, Melander A. Variation in antibiotic use in the European Union. A. Lancet 2001; 357: 1851-1853.

h t t p://www.thelancet.com/journals/lancet/article/ PIIS0140-6736(00)04972-2/fulltext

7. Moynihan R, Heath I, Henry D. Selling sickness: the pharmaceutical industry and disease mongering. BMJ 2002; 324: 886-891.

http://www.bmj.com/cgi/content/full/324/7342/886

8. GöтzсHE PC. Commentary: Medicalisation of risk factors. BMJ 2002; 324-890. http://www.bmj.com/cgi/reprint/324/7342/886

9. ANGEL M. Relationships with the drug industry: Keep at arm's length BMJ 2009; 338: 328-329.

http://www.bmj.com/cgi/content/full/338/feb03_2/b222

10. VICENS C, Fiol F. Abordaje de la deshabituación a benzodiacepinas en atención primaria. Infor Ter Sist Nac Salud 2008; 32: 52-57.

http://www.msc.es/biblioPublic/publicaciones/docs/vol32_2DeshabBenzo diaAtenPrimaria.pdf

11. Indicators of quality prescribing in Australian general practice: a manual for users. February 2006. National Prescribing Service (Australia). Published Surry Hills, N.S.W: National Prescribing Service, 2006.

http://catalogue.nla.gov.au/Record/3697314?lookfor=author:"National\%20 Prescribing\%20Service\%20(Australia)" \&offset=1\&max=10

12. Weingart SN, Toth M, Sands DZ, Aronson MD, Davis RB, Phillips RS. Physician's decisions to override computerized drug alerts in primary care". Arch Intern Med 2003; 163: 2625-2631.

http://archinte.ama-assn.org/cgi/content/abstract/163/21/2625

13. Gurwitz JH, Field TS, Rochon P, Judge J, Harrold LR, Bell CM et al. Effect of computerized provider order entry with clinical decision support on adverse drug events in the long-term care setting. J Am Geriatr Soc [resumen]. 2008; 56: 2225-2233.

http://www3.interscience.wiley.com/journal/121475526/abstract?CRETRY= $1 \&$ SRETRY $=0$ 
14. Kaboli PJ, McClimon BJ, Hoth MAB, Barnett MJ. Assessing the accuracy of computerized medication histories. Am J Manag Care 2004; 1: 872-877.

http://www.ajmc.com/article.cfm?ID=2762\&CFID=15952263\&CFTOKE $\mathrm{N}=72974555$

15. Domínguez S. Más receta o más electrónica. AMF 2009; 5: 2-3.

http://www.amf-semfyc.com/revistas/secciones/index.php?Mg\%3D\%3D

16. Avery AJ, JAMES V. Developing nurse prescribing in the UK. BMJ 2007: 335; 316.

http://www.bmj.com/cgi/content/extract/331/7526/1154

17. LAPORTE JR, Tognoni G, editors. Principios de epidemiología del medicamento. Barcelona: Masson-Salvat; 1993. http://www.icf.uab.es/pem/llibre.htm 